\title{
Estado nutricional e condições de saúde da equipe de enfermagem
}

\author{
Nutritional status and health conditions of the nursing team \\ Estado nutricional y condiciones de salud del equipo de enfermería
}

Recebido: 09/08/2021 | Revisado: 15/08/2021 | Aceito: 17/08/2021 | Publicado: 19/08/2021

\author{
Valdinete Mendes Da Silva \\ ORCID: https://orcid.org/0000-0003-3473-0207 \\ Universidade do Estado de Mato Grosso, Brasil \\ E-mail: valdinete.mendes@unemat.br \\ Julia De Souza Alves \\ ORCID: https://orcid.org/0000-0001-5085-5646 \\ Universidade do Estado de Mato Grosso, Brasil \\ E-mail: julia.alves@unemat.br \\ Grasiela Cristina da Silva Botelho Silvestre \\ ORCID: https://orcid.org/0000-0001-5367-4648 \\ Universidade do Estado de Mato Grosso, Brasil \\ E-mail: grasiela.silvestre@unemat.br \\ Roseany Patrícia Silva Rocha \\ ORCID: https://orcid.org/0000-0002-2295-5321 \\ Universidade do Estado de Mato Grosso, Brasil \\ E-mail: roseany.rocha@unemat.br \\ Fayanne Araujo Gaiva \\ ORCID: https://orcid.org/0000-0002-2851-0710 \\ Universidade do Estado de Mato Grosso, Brasil \\ E-mail: fayaraujo@hotmail.com
}

\begin{abstract}
Resumo
Objetivo: Descrever o perfil sóciodemográfico, as medidas antropométricas e as condições de saúde dos profissionais de enfermagem que atuam nas Estratégias Saúde da Família. Metodologia: Trata-se de um estudo transversal, descritivo e de abordagem quantitativa, realizado com profissionais da enfermagem das Estratégias de Saúde da Família de um município do médio norte de Mato Grosso. Resultado: O estudo foi estruturado por meio da análise dos 32 questionários respondidos pelos profissionais de enfermagem das ESF's. A coleta de dados se deu por intermédio de questionários Resultados: O estudo foi estruturado por meio da análise dos 32 questionários respondidos pelos profissionais de enfermagem das ESF's. Observou-se que a maioria eram do sexo feminino de cor/raça parda. Quanto a idade destacamse as faixas etárias de 27 a 48 anos. O tempo de atuação prevaleceu de 3 a 13 anos, quanto a renda familiar verificou o maior percentual de 3 a 4 salários mínimos. Quanto aos dados da medidas antropométricas e condições de saúde dos profissionais de enfermagem apresentou que 15 dos entrevistados estão com sobrepeso/pré-obesos. Com relação a Circunferência da Cintura predominou o risco muito elevado para obesidade. Conclusão: Os dados encontrados demonstram que o maior índice dos profissionais de enfermagem estão com sobrepeso/pré-obesos. Ao mensurar o estado nutricional dos profissionais, evidencia-se a relevância e importância de se avaliar tal variável visando a promoção e prevenção de futuros agravos a saúde e complicações ao indivíduo.
\end{abstract}

Palavras-chave: Estado nutricional; Obesidade; Enfermagem; Sobrepeso; Doenças crônicas não transmissíveis.

\begin{abstract}
Objective: To describe the sociodemographic profile, anthropometric measurements and health conditions of nursing professionals working in Family Health Strategies. Methodology: This is a cross-sectional, descriptive study with a quantitative approach, carried out with nursing professionals from the Family Health Strategies of a municipality in the middle north of Mato Grosso. Results: The study was structured through the analysis of 32 questionnaires answered by the nursing professionals of the ESF's. Data collection took place through questionnaires. Results: The study was structured through the analysis of 32 questionnaires answered by the nursing professionals of the ESF's. It was observed that most were females of brown color/race. As for age, the age groups from 27 to 48 years are highlighted. The length of work prevailed from 3 to 13 years, while family income verified the highest percentage of 3 to 4 minimum wages. As for the data on anthropometric measurements and health conditions of nursing professionals, it was shown that 15 of the respondents are overweight/pre-obese. Regarding the waist circumference, a very high risk for obesity predominated. Conclusion: The data found show that the highest rate of nursing professionals are overweight/pre-obese. When measuring the nutritional status of professionals, the relevance and importance of evaluating this variable is evidenced, aiming at the promotion and prevention of future health problems and complications to the individual.
\end{abstract}

Keywords: Nutritional status; Obesity; Nursing; Overweight; Noncommunicable diseases. 


\begin{abstract}
Resumen
Objetivo: Describir el perfil sociodemográfico, las medidas antropométricas y las condiciones de salud de profesionales de enfermería que trabajan en Estrategias de Salud de la Familia. Metodología: Se trata de un estudio descriptivo transversal con enfoque cuantitativo, realizado con profesionales de enfermería de las Estrategias de Salud de la Familia de un municipio del centro norte de Mato Grosso. Resultado: El estudio se estructuró a través del análisis de 32 cuestionarios respondidos por los profesionales de enfermería de la ESF. La recolección de datos se realizó a través de cuestionarios Resultados: El estudio se estructuró a través del análisis de 32 cuestionarios respondidos por los profesionales de enfermería de la ESF. Se observó que la mayoría eran hembras de color / raza marrón. En cuanto a la edad, se destacan los grupos de edad de 27 a 48 años. La duración del trabajo predominó de 3 a 13 años, mientras que el ingreso familiar registró el mayor porcentaje de 3 a 4 salarios mínimos. En cuanto a los datos sobre medidas antropométricas y condiciones de salud de los profesionales de enfermería, se demostró que 15 de los encuestados tienen sobrepeso / pre-obesidad. En cuanto a la circunferencia de la cintura, predominó un riesgo muy alto de obesidad. Conclusión: Los datos encontrados muestran que la mayor tasa de profesionales de enfermería tiene sobrepeso / preobesidad. Al medir el estado nutricional de los profesionales, se evidencia la relevancia e importancia de evaluar esta variable, apuntando a la promoción y prevención de futuros problemas de salud y complicaciones para el individuo.
\end{abstract}

Palabras clave: Estados nutricionales; Obesidad; Enfermería; Exceso de peso; Enfermedades no transmisibles.

\title{
1. Introdução
}

A Estratégia Saúde da Família (ESF), é uma unidade de saúde primária que tem como objetivo contribuir para o aprimoramento e consolidação do Sistema Único de Saúde (SUS) e proporcionar a qualidade de vida da população brasileira intervindo nos fatores de risco, como a falta de atividade física, má alimentação, tabagismo, entre outros (Barbine, Nora \& Schaerfer, 2016; Brasil, 2020).

Como parte constituinte do trabalho na ESF temos o médico generalista, enfermeiro, técnicos de enfermagem, agentes comunitários de saúde, dentista, auxiliar de saúde bucal, auxiliar de serviços gerais e administrativo (Peruzzo et al., 2018). Assim, enquanto membros da equipe multiprofissional, o enfermeiro e técnico de enfermagem, assumem um papel importante na promoção, proteção, recuperação da saúde e aos preceitos éticos no que se refere aos cuidados da saúde e bem-estar da população, e da coletividade (Brasil, 2017; Peruzzo et al., 2018).

Com a evolução desta profissão como ciência e prática social, o enfermeiro passou a apropriar-se de papeis não só na assistência, mas também na liderança, pesquisa e incorporando a formação profissional conhecimentos de outros saberes, como a ciência da administração. Neste contexto, a enfermagem vem sofrendo modificações na dimensão do seu processo de trabalho, vivenciando uma rotina estressante, onde tem ocasionado desgaste, cansaço e sobrecarga (Freire \& Costa, 2016; Siqueira et al., 2019).

Devido aos riscos prejudiciais à saúde e o aumento significativo da prevalência nos últimos anos, o sobrepeso e a obesidade se tornou um dos problemas mundiais de saúde pública segundo a Organização Mundial de Saúde (OMS) (Brasil, 2019). É uma doença crônica de origem multifatorial sendo caracterizada pelo excesso de gordura corporal, onde os riscos aumentam consideravelmente quando o índice de massa corporal (IMC) ultrapassa a 25kg/m (Barbine, Nora \& Schaerfer, 2018; Souza et al., 2018).

Dados atuais no país, indicam que o índice de brasileiros que atingiu a obesidade nos últimos treze anos aumentou cerca de 67,8\%, havendo um progresso entre os adultos da faixa etária de 25 a 44 anos de idade, com 84,2\% (Brasil, 2019). O excesso de peso e/ou a obesidade em profissionais de saúde é uma condição que pode gerar consequências ao indivíduo, a sociedade e aos serviços de saúde e, por isso, a necessidade de estratégias para a promoção da saúde dentro do ambiente de trabalho (Domingues, Silva \& Bierhals, 2019; Souza et al., 2018; Pereira et al., 2017).

Em face ao exposto, esse estudo tem como objetivo descrever o perfil sociodemográfico, a avaliação antropométrica e as condições de saúde dos profissionais de enfermagem que atuam nas Estratégias de Saúde da Família. 


\section{Metodologia}

Trata-se de um estudo descritivo, transversal com abordagem quantitativa relacionado ao perfil sociodemográfico, condições de saúde e medidas antropométricas dos profissionais de enfermagem (Almeida Filho \& Barreto, 2012). Foi realizado nas 10 (dez) Estratégias de Saúde da Família (ESF) de um município do medio-norte de Mato Grosso- MT.

A população do estudo foi composta por trinta e dois (32) profissionais de enfermagem, sendo nove (09) enfermeiros e vinte e três (23) técnicos de enfermagem. Para definição da amostra, aplicou-se os critérios de inclusão: atuação nas ESF e como critério de exclusão considerou os profissionais que estavam de férias, folga ou em afastamento no momento da coleta dos dados. Não houve recusa de nenhum profissional.

A coleta de dados foi realizada nos meses de fevereiro e março do ano de 2020, por meio de aplicação de um questionário que foi realizada por entrevista. O instrumento foi disposto em perfil sociodemográfico, condições de saúde e medidas antropométricas.

Foi realizado previamente uma reunião com todos os profissionais de enfermagem, para esclarecer os objetivos da pesquisa, finalidade da avaliação do estado nutricional, os aspectos éticos da pesquisa seguido das orientações sobre o TCLE.

Após o aceite de cada participante, deu-se início a entrevista individual e posteriormente foi realizado a verificação da aferição da pressão arterial seguida da coleta dos dados antropométricos que incluem: peso (kg), altura (centímetro) e circunferência da cintura. Para avaliação dos dados antropométricos foi utilizada uma balança antropométrica, fita métrica flexível de $200 \mathrm{~cm}$, estetoscópio, esfigmomanômetro e calculadora para o cálculo do índice de massa corporal (IMC) e circunferência da cintura (CC), disponível em cada unidade.

Os dados coletados manualmente foram transportados e digitados para planilhas eletrônicas em um banco de dados do programa Excel 2019. As variáveis numéricas foram descritas por estatística descritiva, por medidas de tendência central e dispersão. As variáveis categóricas foram apresentadas por frequência absoluta e relativa.

Para definição da análise do perfil antropométrico foi utilizado as fórmulas para o cálculo do IMC e valores de referência de acordo com a OMS (2004), para pessoas com idade igual ou acima de 18 anos. O índice de massa corporal é caracterizado como a divisão do peso pela altura elevada ao quadrado, desta forma o peso corporal é expresso em $\mathrm{kg}$ e a altura em $\mathrm{m}^{2}$ (Pohl et al., 2018).

O estudo cumpriu com as exigências que regem as pesquisas com seres humanos de acordo com a Resolução de nº466/2012, sendo aprovado pelo Comitê de Ética em Pesquisa da UNEMAT, sob o número da CAAE: 17865219.8.0000.5166.

\section{Resultados e Discussão}

O estudo foi estruturado por meio da análise de 32 questionários respondidos pelos profissionais de enfermagem das ESF's. Conforme demonstrado, obteve-se a predominância de técnicos em enfermagem de 23 (71,88\%), enquanto os enfermeiros foram 9 (28,12\%). Observou-se que a maioria eram do sexo feminino 27 (84,37\%) e se autodeclararam da raça/cor parda 25 (78,12\%). Quanto a idade destaca-se as faixas etárias de 27 a 37, 9 (28,13\%) e de 38 a 48 anos 9 (28,13\%). Com relação ao tempo de atuação prevaleceu de 3 a 13 anos 10 (31,25\%), quanto a renda familiar dos profissionais de enfermagem verificou o maior percentual de 3 a 4 salários mínimos 12 (37,5\%) (Tabela 1). 
Tabela 1 - Distribuições das características sociodemográficas dos profissionais de enfermagem do município de Diamantino, MT, Brasil, 2020. $(n=32)$.

\begin{tabular}{llll}
\hline Variáveis & & $N$ & $\%$ \\
\hline Atuação & Enfermeiros & 9 & 28,12 \\
& Técnicos de enfermagem & 23 & 71,88 \\
Sexo & Masculino & & 15,63 \\
& Feminino & 5 & 84,37 \\
Faixa etária & 27 a 37 & 27 & 28,13 \\
& 38 a 48 & 9 & 28,13 \\
& 49 a 59 & 9 & 21,87 \\
Anos de atuação & 60 ou mais & 7 & 6,25 \\
& 3 a 13 & 2 & 31,25 \\
& 14 a 24 & 10 & 28,13 \\
& 25 ou mais & 9 & 18,75 \\
& 1 a 2 salários mínimos & 6 & 31,25 \\
\hline
\end{tabular}

*SM - Salário Mínimo vigente (1.045,00 reais). Fonte: Autores.

Quanto aos dados antropométricos e condições de saúde dos profissionais de enfermagem, apresenta que 15 (46,87\%) dos entrevistados estão com sobrepeso/pré-obesos. Com relação a CC predominou o risco muito elevado para a obesidade destacando14 $(43,75 \%)$ profissionais.

Adiante, os dados da Tabela 2 ilustram as Doenças Crônicas Não Transmissíveis, a prevalência de participantes que não apresentava nenhuma DCNT de 24 (75\%), Hipertensão Arterial Sistêmica 4 (12,5\%) e HAS e DM 4 (12,5\%). Quanto a atividade física a porcentagem de pessoas que praticam a mesma raramente é $19(59,38 \%)$. Referente ao consumo de bebidas, o consumo de café prevaleceu em 30 (93,75\%), seguida do consumo de bebidas alcoólicas 17 (53,13\%) e refrigerante 14 (43,75\%). 
Tabela 2 - Distribuição dos dados antropométricos e condições de saúde de acordo com o sexo dos profissionais de enfermagem do município de Diamantino, MT, Brasil, 2020. $(n=32)$.

\begin{tabular}{|c|c|c|c|c|c|c|}
\hline \multirow[t]{2}{*}{ Variáveis } & \multirow[b]{2}{*}{$n$} & \multirow[b]{2}{*}{$\%$} & \multicolumn{2}{|c|}{ Feminino } & \multicolumn{2}{|c|}{ Masculino } \\
\hline & & & $N$ & $\%$ & $n$ & $\%$ \\
\hline \multicolumn{7}{|l|}{ IMC } \\
\hline Faixa normal & 11 & 34,38 & 10 & 31,25 & 1 & 3,12 \\
\hline Sobrepeso/Pré-obeso & 15 & 46,87 & 13 & 40,63 & 2 & 6,25 \\
\hline Obeso classe I & 4 & 12,5 & 2 & 6,25 & 2 & 6,25 \\
\hline Obeso classe II & 2 & 6,5 & 2 & 6,25 & 0 & 0 \\
\hline \multicolumn{7}{|l|}{$\mathrm{CC}$} \\
\hline Normal & 7 & 21,87 & 6 & 18,75 & 1 & 3,12 \\
\hline Risco Elevado & 11 & 34,38 & 9 & 28,13 & 2 & 6,25 \\
\hline Risco Muito Elevado & 14 & 43,75 & 12 & 37,5 & 2 & 6,25 \\
\hline \multicolumn{7}{|c|}{ Doenças crônicas não transmissíveis } \\
\hline Hipertensão Arterial Sistêmica & 4 & 12,5 & 3 & 9,37 & 1 & 3,13 \\
\hline HAS e DM & 4 & 12,5 & 4 & 12,5 & 0 & 0 \\
\hline Sem HAS e DM & 24 & 75 & 20 & 62,5 & 4 & 12,5 \\
\hline \multicolumn{7}{|l|}{ Atividade física } \\
\hline Raramente & 19 & 59,38 & 15 & 46,88 & 4 & 12,5 \\
\hline 1 a 2 vezes por semana & 5 & 15,62 & 5 & 15,62 & 0 & 0 \\
\hline Acima de 3 vezes por semana & 8 & 25 & 7 & 21,87 & 1 & 3,13 \\
\hline \multicolumn{7}{|l|}{ Consumo de bebidas } \\
\hline Refrigerante & 14 & 43,75 & 10 & 31,25 & 4 & 12,5 \\
\hline Café & 30 & 93,75 & 25 & 78,13 & 5 & 15,63 \\
\hline Bebidas alcoólicas & 17 & 53,13 & 13 & 40,63 & 4 & 12,49 \\
\hline
\end{tabular}

Fonte: Autores.

\section{Discussão}

No presente estudo, foi discutido o estado nutricional associado ao perfil sociodemográfico, condições de saúde e dados antropométricos dos profissionais de enfermagem das Estratégias Saúde da Família.

Segundo pesquisas nacionais o quantitativo de técnicos de enfermagem é maior que de Enfermeiros se assemelhando ao do presente estudo, devido ao dimensionamento do pessoal de enfermagem, da demanda de empregos, do curto período de estudos para a formação (Pinhatti et al., 2018).

O gênero feminino foi predominante na equipe de enfermagem, demostrando que a feminilização na área da enfermagem tem permanecido por décadas devido razão tradicional e cultural. Tal resultado se assemelha aos resultados de outros estudos o que também pode estar relacionado com a luta da população feminina no mercado de trabalho (Araújo et al., 2017; Ramos et al., 2017).

Em relação a raça/cor a predominância da pesquisa foi a parda, evidenciando conciliação com a pesquisa realizada pelo IBGE, onde 46,8\% dos brasileiros se autodeclaram pardos, 42,7\% como brancos, 9,4\% como pretos e 1,1\% como amarelos ou indígenas (Brasil, 2019). Em contrapartida, dados de uma pesquisa sobre o perfil da equipe de enfermagem no Brasil apresentaram que $41,5 \%$ se autodeclararam da cor parda o que corrobora com esse estudo (Pinhatti et al., 2017). 
Quanto a idade, os resultados apontam que os profissionais de enfermagem estão entre a faixa etária de 27 a 48 anos, sendo uma população relativamente jovem, resultado este que se assemelha a outros estudos realizados com essa população (Peruzzo et al., 2018; Pinhatti et al., 2017).

Com relação ao tempo de atuação nas ESFs, os resultados demonstraram que os profissionais atuaram em média de 3 a 13 anos (31,25\%). Ao levar este assunto para o trabalho de enfermagem, percebe-se que outros estudos alguns profissionais relataram que atuaram ao menos entre 5 a 15 anos na profissão de enfermagem (Domingues, Silva \& Bierhals, 2019; Ramos et al., 2017).

Quanto a renda familiar dos profissionais de enfermagem verificou-se a média de 3 a 4 salários mínimos, visto que o valor do piso salarial vigente em 2020 é de R \$1,045.00 segundo o Ministério da Economia, e que também vai de encontro com os resultados de outros estudos (Pohl et al., 2018).

Sobre os dados antropométricos e condições de saúde as informações do IMC apontam que 15 (46,87\%) dos entrevistados estão com sobrepeso/pré-obesos com o valor do IMC entre 25 a 29,9 de acordo com a mensuração. Os dados apresentados são semelhantes a outras pesquisas que apresentam que os profissionais de enfermagem estavam acima do peso (Malta et al., 2017; Pereira et al., 2017; Siqueira et al., 2019).

Relatos que há prevalência de 30,9\% de indivíduos com sobrepeso em 2006, e de 33,2\% de sobrepesos em 2010 na população adulta residente em todos estados do Brasil, incluindo o Distrito Federal (Malta et al., 2017; Siqueira et al., 2019). Desta forma é válido observar que a porcentagem de indivíduos com excesso de peso vem crescendo anualmente de acordo com as características sócio demográficas (Freitas et al., 2016).

A variável circunferência da cintura (CC), a qual mostrou um risco muito elevado para obesidade 14 (43,75\%). Outras pesquisas corroboram com esse resultado afirmando que 49,7\% dos trabalhadores de enfermagem que atuavam em um hospital público de nível terciário situado em Fortaleza-Ceara estavam com a CC acima da média permitida, já que a média ideal é de $<80$ cm para mulheres e $<94 \mathrm{~cm}$ para homens (Pereira et al., 2017; Siqueira et al., 2019).

O excesso de gordura abdominal contribui para o aumento do risco de doenças cardiovasculares e DCNT. A combinação do IMC e CC ambos alterados, podem aumentar consideravelmente as chances de adquirir uma DCNT (Domingues, Silva \& Bierhals, 2019; Freitas et al., 2016; Pereira et al., 2017).

Conforme a pesquisa de Domingues, Silva e Bierhals (2019), que buscou correlacionar os dados antropométricos (IMC e CC) de 80 profissionais de enfermagem do Rio Grande do Sul, foi identificado que esses estavam com sobrepeso e alguns apresentavam obesidade, foi estimada em 56\% o risco para doenças cardiovasculares.

Em relação as DCNT, foi verificado que 24 (75\%) dos participantes não possuíam nenhum tipo de DCNT, porém 4 $(12,5 \%)$ dos integrantes relataram ter Hipertensão Arterial Sistêmica (HAS) e 4 12,5\% Diabetes Mellitus (DM). Estudos indicam, que pelo menos um terço da população Brasileira possui algum tipo de DCNT (Pereira et al., 2017).

Seguindo resultados semelhantes a literatura, Pinhatti et al. (2017), corroborou com estudo supracitados, onde existe uma alta prevalência de DCNT entre os profissionais de enfermagem sendo aguçados pelas exaustivas cargas de trabalho. Dito isso dificultando na disponibilidade para gozar de práticas regulares de atividades físicas, tempo de lazeres, boa alimentação e aproveitar de descanso adequado, fatores estes que influenciam para o desenvolvimento e o agravamento de patologias como a hipertensão arterial sistêmica (HAS) e diabetes mellitus (DM) (Domingues, Silva \& Bierhasls, 2019; Pinhatti et al., 2017; Pereira et al., 2017).

Relativo à prática de atividade física, observou-se no estudo que 19 (59,39\%) dos profissionais praticavam exercícios físicos raramente. Pesquisas apontam que aproximadamente 50\% dos trabalhadores de enfermagem não praticam atividades físicas alegando falta de tempo (Bottcher, 2019; Freire \& Costa, 2016). 
A inatividade física está relacionada com o índice de mortalidade, sendo o quarto fator de risco para mortalidade precoce por DCNT, tais achados são preocupantes tendo em vista que a prática regular de atividade física vai de encontro com a promoção de saúde, prevenção de doenças além de trazer benefícios para a saúde física, mental e social dos indivíduos, propiciando também liberdade de locomoção, interação social e lazer (Freire \& Costa, 2016; Ramos et al., 2017).

Em relação a ingestão de bebidas diversas, $30(93,75 \%)$ dos profissionais fazem uso continuo do café, seguido do consumo de bebidas alcoólicas 17 (53,13\%) e de refrigerante 14 (43,75\%).

O presente estudo ilustrou relação positiva entre o consumo de álcool e o sobrepeso. O consumo de bebidas alcoólicas deste estudo foi parecido com outros estudos onde pode-se observar uma porcentagem elevada de profissionais de saúde que faz ingestão do mesmo (Junqueira et al., 2017; R. Souza et al., 2018; L. Souza et al., 2020).

Estudos de Junqueira et al. (2017), evidenciou que o álcool possui valor energético, e tem a habilidade de suprimir as necessidades calóricas de um indivíduo e/ou levá-lo ao sobrepeso, dependendo da quantidade, frequência e modo de consumo.

Considerando os fatores ligados ao estilo de vida na etiologia do sobrepeso e obesidade e doenças cardiovasculares, avalia-se o sedentarismo, o consumo de bebidas alcoólicas e o consumo de refrigerantes (Bezerra \& Alencar, 2018). O consumo de grandes porções de bebida alcoólica está associado ao aumento da pressão arterial e da mortalidade cardiovascular em geral (Chaves et al., 2018).

A pesquisa apresentou um elevado consumo de refrigerante pelos participantes, segundo estudos a epidemia de sobrepeso e obesidade aumentou consideravelmente após o refrigerante se tornar popular nos Estados Unidos quanto na Europa, tendo em vista que também houve um aumento de porções consumidas (Chaves et al., 2018; Medeiros et al., 2016).

Por fim, de acordo com autores o tamanho da porção de refrigerante e bebida alcoólica mostrou associação positiva com o excesso de peso, já que, uma vez que aumenta o consumo dos mesmos, também a um aumento excessivo de calorias e consequentemente desencadeia o sobrepeso (Freitas et al., 2016; Junqueira et al., 2017).

Desta maneira, tendo em vista as limitações, seus resultados não podem ser generalizados, pois apresentam suas particularidades envolvendo os profissionais de enfermagem em um contexto regional. Entretanto, apresenta subsídios para pensar a discussão do tema de forma mais efetiva, considerando-se as necessidades, limitações e experiências vivenciadas diante dessa temática. Outra limitação é inerente ao próprio desenho do estudo, ou seja, a causalidade reversa.

No presente estudo, observou-se associações entre condições socioeconômicas, categoria profissional e contexto cultural. Apresentando um maior percentual de sobrepeso/obesidade em técnicos de enfermagem.

\section{Conclusão}

Conclui-se com os dados encontrados demonstram que o maior índice dos profissionais de enfermagem estão com sobrepeso/pré-obesos. Ao mensurar o estado nutricional dos profissionais, evidencia-se a relevância e importância de se avaliar tal variável visando a promoção e prevenção de futuros agravos a saúde e complicações ao indivíduo.

Os resultados obtidos por meio desta pesquisa propõem o fortalecimento e ampliação dos processos de educação permanente e apoio técnico a equipe de enfermagem, para instigar esses indivíduos a aderir um estilo de vida saudável e praticar a mudança de comportamento, estilo de vida e alimentar.

As informações aqui expostas podem subsidiar a implementação de programas eficazes de promoção da saúde no local de trabalho, objetivando a prevenção da obesidade e outras doenças crônicas não transmissíveis (DCNT), combinando-se com estratégias de intervenção focadas na equipe de enfermagem.

Espera-se, diante do exposto a suma importância que com esta pesquisa possa surgir novos estudos que vise e amplie os cuidados com os profissionais de enfermagem, subsidiando intervenções como educação continuada que agem diretamente 
na má alimentação e a inatividade física, com o objetivo de promover saúde entre esses profissionais que são o alicerce do cuidado.

\section{Referências}

Almeida Filho, N., \& Barreto, M.L. (2012). Epidemiologia e Saúde: Fundamentos, Métodos, Aplicações. Editora Guanabara Koogan.

Araujo, A. N. A., Filho Lunardi, W. D., Alvarenga, M. R. M., Oliveira, R. D., Souza, J.C., \& Vidamantas, S. (2017). Perfil sociodemografico dos enfermeiros da rede hospitalar. Rev enferm UFPE on line. 11(11):4716-25.

Barbiani, R., Nora C. R. D., \& Schaerfer R. (2016). Praticas do enfermeiro no contexto da atenção básica: scoping review. Rev. Latino-Am. Enfermagem. 24: $1-12$.

Bezerra, I. N., \& Alencar E. S. (2018). Associação entre excesso de peso e tamanho das porções de bebidas consumidas no Brasil. Revista Saúde Pública. 52:21.

Bottcher, L. B. (2019). Atividade física como ação para promoção da saúde: Um ensaio critico. Revista Eletrônica Gestão e Saúde. $2019 ; 1982-4785$.

Brasil. (2020). Ministério da Saúde. Sistema Únicode Saúde(SUS): estrutura, princípios e comofunciona. <https://www.saude.gov.br/sistema-unico-de-saude >Acesso.

Brasil. (2017). Ministério da Saúde. Estratégia Saúde da Família (ESF). 2017. <https://www.saude.gov.br/acoes-e-programas/saude-da-familia/sobre-oprograma.

Brasil. (2019). Ministério da Saúde. Brasileiros atingem maior índice de obesidade nos últimos treze anos. https://saude.gov.br/noticias/agencia-saude/45612-brasileirosatingem-maior-indice-de-obesidade-nos-ultimos-treze-anos.

Chaves, O.C., Melendez, G. V., Costa, D. A. S., \& Caiaffa. W. T. (2018). Consumo de refrigerantes e índice de massa corporal em adolescentes brasileiros: Pesquisa nacional de saúde do escolar. Rev Bras epidemiol. 21(1):80010.

Domingues, J. G., Silva, B. B. C., \& Bierhals, I. O. (2019). Doenças crônicas não transmissíveis em profissionais de enfermagem de um hospital filantrópico no Sul do Brasil. Epidemiol. Serv. Saúde. 28 (2).

Freire, M. N., \& Costa, E. R. (2016). Qualidade de vida dos profissionais de enfermagem no ambiente de trabalho. Revista Enfermagem Contemporânea. 5(1): $151-158$.

Freitas, P. P., Assunção, A. A., Bassi, I. B., \& Lopes, A. C. S. (2016). Excesso de peso e ambiente de trabalho no setor público municipal. Rev. Nutr. 29(4): 519527.

Junqueira, M. A. B., Ferreira, M. C. M., Soares, G. T., Brito, I. E., Santos, M. A., \& Pillon, S. C. (2017). Uso de álcool e comportamento de saúde entre profissionais da enfermagem. Rev esc Enferm USP.

Malta, C. M., Santos, M. A. S., Andrade, S. S. C. A., Oliveira, T. P., Stopa, S. R., Oliveira, M. M., \& Jaime, P. (2016). Tendência temporal dos indicadores de excesso de peso em adultos nas capitais brasileiras, 2006-2013. Ciência \& Saúde Coletiva. 21(4):1061-1069.

Medeiros, P. A., Silva, L. C., Amarante, I. M., Cardoso, V. G., Mensch, K. M., Naman, M., \& Schimith, M. D. (2016) Condições de saúde entre profissionais da atenção básica em saude do município de Santa Maria - RS. Revista Brasileira de ciências da saúde. $20(2): 115-122$.

Pereira, R. S. F., Gusmão, J. L., Santos, C. A., \& Silva, A. (2017). Obesidade e sobrepeso em trabalhadores da enfermagem de um hospital público em São José dos Campos-SP. Revista Enfermagem. 82 (20).

Peruzzo, H. E., Bega, A. G., Lopes, A. P. A. T., Fernandez, M. C., Haddad, L., Peres, A. M., \& Marcon, S. S. (2018). Os desafios de se trabalhar em equipe na estratégia saúde da família. Esc Anna Nery. 22(4).

Pinhatti, E. D. G., Vannuchi, N. T. O., Tenani, M. N. F., \& Haddad, M. C. L. (2017). Influencias sociodemograficas e laborais na satisfação profissional de enfermeiros em hospital público. Rev enferm UERJ. 25: 14405.

Pohl, H. H., Arnold, E. F., Dummel, K. L., Cerentini, T. M., Reuter, E. M., \& Reckziegel, M.B. (2018) Indicadores antropométricos e fatores de risco cardiovascular em trabalhadores rurais. Rev Bras Med Esporte. 24(1).

Ramos, F. R. S., Barth, P. O., Brito, M. J., Caram, C., Silveira, L. R., Brehmer, L. C. F., Dalmolin, G.L., \& Caçador, B. (2019). Aspectos sociodemograficos e laborais associados ao distresse moral em enfermeiros brasileiros. Acta Paul enferm. 32(4): 406-15.

Siqueira, F. V., Reis, D. S., Souza, R. A. L., Pinho, S., \& Pinho, L. (2019). Excesso de peso e fatores associados entre profissionais de saúde da Estratégia Saúde da Família. Cad. Saúde colet. 27 (2): 138-145.

Souza, R. F., Rosa, R. S, Picanço, C. M., Junior, E. V. S., Cruz, D. P., Guimarães, F. E. O., \& Boery, R. N. S. O. (2018). Repercussões dos fatores associados á qualidade de vida em enfermeiras de unidade de terapia intensiva. Rev. Salud Pública. 20(4): 453-459.

Souza, L. P. S., Miranda, A. E. S., Hermsdorff, H. H. M., Silva, C. S. O., Barbosa, D. A., Bressan, J., \& Pimenta, A. M. (2020). Consumo pesado episódio de álcool e excesso de peso em adultos brasileiros - Projeto CUME. Ver Bras Enferm. 73(1): 20190316. 